\title{
Primary nasopharyngeal tuberculosis: About 5 cases
}

\author{
Ballage Amine*, Mkhatri A, Baghdad M, Chaouki A, Rouadi S, Abada R, Roubal M and Mahtar M \\ Department of ENT, 20 Aout Hospital, Ibn Rochd University Hospital, Casa-blanca, Morocco
}

\begin{abstract}
Nasopharyngeal tuberculosis is a rare entity of extrapulmonary tuberculosis. We describe here 5 cases of primarily tuberculosis presented in ENT outpatient department of 20 August Hospital of CHU Ibn Rochd of Casablanca. 5 patients were enrolled in this study ( 3 males and 2 females). The main complaints were nasal obstruction and epistaxis. Clinical examination found no cervical lymphadenopathies and nasal endoscopy found a pale pink mass in all patients. Histological examination confirmed the diagnosis of tuberculosis. All patients showed a complete resolution of the disease after 6 months treatment.
\end{abstract}

\section{Introduction}

Tuberculosis is the leading cause of infectious mortality and continues to be a major challenge to global health [1]. With most frequent site being lungs, nasopharyngeal tuberculosis is a rare type of extrapulmonary tuberculosis comprising only less than $1 \%$ of tuberculosis found in the upper respiratory tract [2].

We are presenting here 5 cases of primary tuberculosis affecting the nasopharynx which is one of rare differential diagnosis of nasopharyngeal mass.

\section{Methods}

We report 5 cases of nasopharyngeal tuberculosis presented in ENT outpatient department of 20 August Hospital of CHU Ibn Rochd of Casablanca between September 2016 and February 2018. The management of these patients was based on nasal endoscopy with biopsy and histological examination and x-ray chest. Epidemiological, clinical, therapeutic and evolutionary parameters were studied. A control by nasal endoscopy was carried out after 6 months of treatment.

\section{Results}

This retrospective descriptive study enrolled 5 patients ( 3 females and 2 males) with mean age of 36 years, range 25-53. No significant past medical or surgical history could be elicited. The main complaints were nasal obstruction and epistaxis. No history of evening rise fever, weight loss, cough or contact with tuberculous patients was noticed. Clinical exam found no cervical lymphadenopathies in all patients. Nasal drip on examination of throat was noticed in 3 patients. Normal tympanic membranes were found in all patients. Nasal endoscopy showed pale pink mass of the nasopharynx in all patients. Biopsy of the nasopharyngeal mass was performed in all patients and unveiled multiple intense chronic inflammatory reaction along with Langerhans giant cells and epithelioid cells. Special stains were employed and showed the presence of acid-fast bacilli.

All patients had normal X-ray chest. Final diagnosis of primary nasopharyngeal was made. The patients were treated by antitubercular drugs for six months as per WHO recommendation. A 6-month followup for all patients was done and revealed normal nasopharyngeal mucosa with good recovery.

\section{Discussion}

Nasopharyngeal lesions have many differential diagnoses including malignancy (squamous cell carcinoma, lymphoma), fungal infection (aspergillosis, mucormycosis), granulomatous inflammation (sarcoidosis, leprosy, syphilis, tuberculosis) and autoimmune disease [3].

Nasopharyngeal tuberculosis is a rare entity, even in endemic tuberculosis areas [4-6]. Two potential pathways of infection have been described. In primary nasopharyngeal tuberculosis, infection occurs directly via nasal ventilation. In secondary disease, infection spreads from site which is mostly lungs, or via haematogenous or lymphogenous spread [7].

The most common symptoms of nasopharyngeal tuberculosis are cervical lymphadenopathy, weight loss, fever, epistaxis, nasal obstruction, hearing loss, otalgia, tinnitus, postnasal drip and night sweats [8]. In the present cases, the main symptoms were epistaxis and nasal obstruction. No cervical lymphadenopathy was found, and no lesion of pulmonary tuberculosis was noticed on $\mathrm{x}$-ray chest.

The diagnosis of nasopharyngeal tuberculosis is based on the histopathological and microbiological findings from biopsy material [9]. Histopathological examination typically reveals granulomatous inflammation with epithelioid giant cells and caseous necrosis. ZiehlNeelsen staining can reveal acid-fast bacilli but biological culture after four to six weeks is more sensitive. The Polymerase Chain Reaction (PCR) test is the gold standard of identifying the tuberculous bacilli [10]. With oral samples, sensitivity increase from 2 to $17 \%$ on culture to $89-100 \%$ on PCR. In our cases, PCR have not been used due to its inaccessibility.

The treatment of nasopharyngeal tuberculosis is anti-tuberculous triple therapy including isoniazid, rifampicin and ethambutol, or quadritherapy with pyrazinamide for at least six months [6].

${ }^{\star}$ Correspondence to: Ballage Amine, Department of ENT, 20 Aout Hospital, Ibn Rochd University Hospital, Casa-blanca, Morocco, Tel: +212-522-481-010; E-mail: amineballage19@gmail.com

Key words: tuberculosis, nasal endoscopy, cervical lymphadenopathies

Received: October 29, 2018; Accepted: November 16, 2018; Published: November 20, 2018 
When treated correctly, nasopharyngeal tuberculosis carries an excellent prognosis, and complete resolution of disease is the rule [4].

\section{Conclusion}

Nasopharyngeal mass should be nasopharyngeal carcinoma until otherwise. Histological examination of multiple (and sometimes repeated) biopsies is needed both to exclude malignant mass and to confirm tuberculosis. PCR can be very helpful when tuberculosis is strongly suspected, and the culture is negative.

\section{References}

1. Fauci AS (2018) Addressing the Tuberculosis Epidemic: 21st Century Research for an Ancient Disease. JAMA 320: 1315-1316. [Crossref]

2. Patil C, Kharat Patil R, Deshmukh P, Biswas J, John B (2013) Primary tuberculosis of nasopharynx (adenoid)- a rare presentation. Asian Pac J Trop Med 6: 246-248. [Crossref]

3. Sithinamsuwan P, Sakulsaengprapha A, Chinvarun Y (2005) Nasopharyngeal tuberculosis: a case report presenting with diplopia. J Med Assoc Thail Chotmaihet Thangphaet 88: 1442-1446. [Crossref]
4. Prstacic R, Jurlina M, Zizic-Mitrecic M, Janjanin S (2011) Primary nasopharyngeal tuberculosis mimicking exacerbation of chronic rhinosinusitis. J Laryngol Otol 125: 747-749. [Crossref]

5. Sawada N, Inokuchi G, Komatsu H, Kurakawa S, Tada K, et al. (2013) Nasopharyngeal tuberculosis. J Infect Chemother 19: 1158-1160. [Crossref]

6. Tse GM, Ma TK, Chan AB, Ho FN, King AD, et al. (2003) Tuberculosis of the nasopharynx: a rare entity revisited. Laryngoscope 113: 737-740. [Crossref]

7. al Serhani AM, al Rikabi AC, Assiry MA (1997) Primary nasopharyngeal tuberculosis: case report and review of the literature. Ear Nose Throat J 76: 41-42. [Crossref]

8. Waldron J, Van Hasselt CA, Skinner DW, Arnold M (1992) Tuberculosis of the nasopharynx: clinicopathological features. Clin Otolaryngol Allied Sci 17: 57-59. [Crossref]

9. King AD, Ahuja AT, Tse GMK, van Hasselt ACA, Chan ABW (2003) MR imaging features of nasopharyngeal tuberculosis: report of three cases and literature review. AJNR Am J Neuroradiol 24: 279-282. [Crossref]

10. Eguchi J, Ishihara K, Watanabe A, Fukumoto Y, Okuda K (2003) PCR method is essential for detecting Mycobacterium tuberculosis in oral cavity samples. Oral Microbiol Immunol 18: 156-159. [Crossref]

Copyright: (C2018 Amine B. This is an open-access article distributed under the terms of the Creative Commons Attribution License, which permits unrestricted use, distribution, and reproduction in any medium, provided the original author and source are credited. 